\title{
Gênero, Idade, Nível Social e Fatores de Risco Cardiovascular: Considerações Sobre a Realidade Brasileira
}

\author{
Gender, Age, Social Level and Cardiovascular Risk Factors: Considerations on the Brazilian Reality
}

\author{
Carlos Scherr ${ }^{1,2}$ e Jorge Pinto Ribeiro 3,4 \\ Universidade Gama Filho ${ }^{2}$, Rio de Janeiro, RJ; Instituto do Coração e do Diabetes², Rio de Janeiro, RJ; Serviço de Cardiologia do Hospital de Clínicas \\ de Porto Alegre ${ }^{3}$, Porto Alegre, RS; Faculdade de Medicina da Universidade Federal do Rio Grande do Sul', Porto Alegre, RS, Brasil
}

Dados recentes mostram a diminuição da mortalidade por doença aterosclerótica coronária (DAC) nos Estados Unidos. Estima-se que, entre os anos de 1980 e 2000, quase 150.000 mortes foram evitadas (em torno de $44 \%$ do total) devido ao controle de fatores de risco (FR), como hipercolesterolemia, hipertensão arterial sistólica, tabagismo e sedentarismo ${ }^{1}$. Pelos dados do estudo AFIRMAR (estudo de avaliação dos fatores de risco associados com o infarto do miocárdio no Brasil), também se verifica que, no Brasil, a maioria dos FR para infarto agudo do miocárdio (IAM) são passíveis de prevenção².

Também foi comprovada a associação entre a presença de FR na adolescência e até na meia idade, além do surgimento, no futuro, de placas ateroscleróticas nas coronárias. Da mesma forma, o controle precoce dos FR parece afetar positivamente o aparecimento de $\mathrm{DAC}^{3}$. Portanto, diagnosticar e combater estes fatores são da maior importância do ponto de vista de saúde pública, pois têm como objetivo fazer frente à mortalidade por doenças cardiovasculares (DCV) no Brasil. Segundo os últimos dados do Ministério da Saúde, as DCV são responsáveis por grande parte da mortalidade dos brasileiros, correspondendo a $31 \%$ do total, sendo proporcionalmente maior nas mulheres em relação aos homens. Se considerada a faixa etária de 40 anos ou mais, este total corresponde quase à soma das três causas seguintes (neoplasia, respiratória e externa $)^{4}$. Em relação ao restante do mundo, estima-se que 2/3 das mortes por DCV ocorram em países em desenvolvimento, como o Brasil, e correspondam ao dobro em relação aos países desenvolvidos ${ }^{5}$. Além disso, foi demonstrado que a DAC tem alto custo: tanto para o governo quanto para o setor privado, o tratamento é comparativamente mais caro que em países desenvolvidos ${ }^{6}$. Neste artigo, discutimos achados de alguns estudos observacionais brasileiros que podem ter implicações em estratégias preventivas.

Existe uma grande influência de fatores biológicos e sociais no perfil de risco dos indivíduos, o que pode levar a uma maior

\section{Palavras chave}

Fatores de Risco, Identidade de Gênero, Idade, Fatores biológicos, Classe Social, Brasil.

\footnotetext{
Correspondência: Carlos Scherr •

Avenida Visconde de Pirajá, 595 Sl. 1204 - Ipanema - 22410-003 - Rio de Janeiro, RJ, Brasil

E-mail: scherr@all.com.br

Artigo recebido em 25/09/08; revisado recebido em 27/11/08; aceito em 06/01/09
}

incidência das DCV em alguns segmentos da sociedade ${ }^{3}$. Em estudo realizado com 343 escolares, constatamos a provável influência do nível social no perfil lipídico. Quando comparados os níveis médios de colesterol dos escolares das instituições particulares de ensino $(171 \mathrm{mg} / \mathrm{dl})$ aos seus similares públicos/filantrópicos $(136 \mathrm{mg} / \mathrm{dl})$, esta diferença foi estatisticamente significativa, o que ficou comprovado para ambos os sexos e tambem para a fração de lipoproteína de baixa densidade $(\mathrm{LDL})^{7}$. Estes resultados suscitaram a hipótese de que as crianças de nível socioeconômico mais baixo "poderiam estar relativamente protegidas". Isso talvez se explique também pelo fato de sua alimentação ser mais dependente da merenda escolar (sob supervisão de nutricionistas), e possivelmente por desenvolverem mais atividade física em suas brincadeiras. Ademais, outro motivo que justifica tais resultados é seu deslocamento ser efetuado a pé, consistindo em atividade mais intensa e constante do que seus pares, que se locomovem habitualmente de carro ou utilizando transportes coletivos.

Seguindo a linha da prevenção primária, avaliamos a presenca de FR no sexo feminino ${ }^{8}$. Foram entrevistadas 419 mulheres, sendo 97 idosas de um asilo, 98 universitárias, 99 médicas e 125 faxineiras que prestavam servicos em hospitais. A idade média foi de 43 anos, seria menor se excluídas as mulheres do grupo após menopausa. A pressão arterial foi aferida e o colesterol dosado. Como esperado, as idosas tinham uma maior prevalência de FR cardiovascular, tais como hipertensão arterial (HA), diabetes, obesidade e hipercolesterolemia (Tabela 1). Porém, foi nas trabalhadoras da limpeza que encontramos as maiores alterações, apesar da faixa etária ter sido semelhante à das médicas e universitárias. Neste grupo, os níveis de colesterol acima de $200 \mathrm{mg} / \mathrm{dl}$ e a presença de HA foram semelhantes aos das idosas, mas o tabagismo, obesidade e sedentarismo foram mais prevalentes em relação aos outros grupos. O diabetes, entretanto, somente prevaleceu em relação às médicas e universitárias. Neste caso, fomos levados a concluir que provavelmente o nível socioeconômico influenciou negativamente na presença de FR para as DCV. Pode-se até especular que, no caso das crianças que não têm recursos próprios, a situação de pobreza as leva a um perfil de risco, mais apropriado às cardiopatias. Quando essas crianças crescem e começam a ter alguma possibilidade de decisão em relação aos seus hábitos de vida, associada à falta de informação, perdem esta possível proteção inicial e passam a correr um risco mais elevado $^{8}$. Este, então, seria outro segmento a necessitar de uma intervenção precoce. 
No caso de indivíduos que foram acometidos por DAC ainda em idade jovem, uma análise retrospectiva de 236 pacientes entre 17 e $45 \operatorname{anos}^{9}$ (média de 38 anos) - sendo $80 \%$ homens e $58 \%$ com história prévia de IAM -, constatouse que $80 \%$ desse total apresentavam três ou mais FR e $58 \%$, quatro ou mais. Excluindo-se fatores subjetivos como estresse, sedentarismo e obesidade, $88 \%$ dos pacientes tinham três ou mais fatores de risco. As alterações mais recorrentes foram tabagismo (75\%), sedentarismo (65\%) e história familiar (60\%). As associações mais frequentes de FR foram as de fumo com diabetes e fumo com hipercolesterolemia ${ }^{10}$. Na situação daqueles que apresentaram a DAC ainda jovens, é provável que a avaliação tenha sido facilitada pela presença de vários destes fatores num mesmo indivíduo, permitindo uma prevenção primária talvez mais eficaz. É necessário lembrar que este grupo costuma desenvolver a doença com mais gravidade, devido à evolução rápida da aterosclerose e sua localização preferencial na artéria descendente anterior, ocorrência que atingiu 67\% de 114 pacientes, internados com história de Síndrome Isquêmica Aguda $(\mathrm{SIA})^{10}$.

No intuito de incluir pacientes de uma faixa etária mais ampla e em maior número, avaliamos, em corte transversal, uma população de 2.337 pacientes coronarianos em tratamento ambulatorial, com idade variando de 26 a 89 anos, sendo $61 \%$ do sexo masculino ${ }^{11}$. Nesta amostra, encontrou-se uma prevalência maior de FR no sexo feminino. O mesmo ocorreu ao avaliarmos os indivíduos com três ou mais fatores (Tabela 2). Quando comparados os pacientes com idade de 55 anos ou menos com os de 65 ou mais, constatamos a presença significativamente maior de história familiar, extabagismo, obesidade e tabagismo atual no primeiro grupo, onde também foi encontrada uma concentração maior de pacientes com três ou mais fatores, em comparação ao segundo grupo (Tabela 3) ${ }^{12}$.

\section{Tabela 1 - Prevalência de fatores de risco em mulheres.}

\begin{tabular}{|c|c|c|c|c|c|}
\hline & $\begin{array}{l}\text { Idosas } \\
(n=97)\end{array}$ & $\begin{array}{l}\text { Universitárias } \\
\qquad(\mathrm{n}=98)\end{array}$ & $\begin{array}{l}\text { Médicas } \\
(\mathrm{n}=99)\end{array}$ & $\begin{array}{c}\text { Faxineiras } \\
(n=125)\end{array}$ & $\mathbf{P}$ \\
\hline Tabagismo & $10 \%$ & $25 \%$ & $8 \%$ & $28 \%$ & $<0,001$ \\
\hline Hipertensão arterial sistêmica & $39 \%$ & $1 \%$ & $6 \%$ & $20 \%$ & $<0,001$ \\
\hline Obesidade & $43 \%$ & $13 \%$ & $17 \%$ & $54 \%$ & $<0,001$ \\
\hline Colesterol >200 mg\% & $28 \%$ & $14 \%$ & $16 \%$ & $21 \%$ & 0,073 \\
\hline Diabetes & $15 \%$ & $2 \%$ & $2 \%$ & $6 \%$ & $<0,001$ \\
\hline Estresse & $52 \%$ & $45 \%$ & $70 \%$ & $58 \%$ & 0,003 \\
\hline Menopausa & $100 \%$ & $9 \%$ & $13 \%$ & $30 \%$ & $<0,001$ \\
\hline Sedentarismo & $81 \%$ & $71 \%$ & $73 \%$ & $88 \%$ & 0,0061 \\
\hline
\end{tabular}

Testes estatísticos utilizados: Qui Quadrado e Teste de Student.

Tabela 2 - Prevalência dos fatores de risco por gênero.

\begin{tabular}{lccc} 
& $\begin{array}{c}\text { Mulheres } \\
\%\end{array}$ & $\begin{array}{c}\text { Homens } \\
\%\end{array}$ & $\mathbf{P}$ \\
\hline Hipertensão & 73 & 54 & $<0,0001$ \\
\hline História familiar & 59 & 50 & $<0,0001$ \\
\hline Ex-tabagismo & 30 & 50 & $<0,0001$ \\
\hline Dislipidemia & 54 & 44 & $<0,0001$ \\
\hline Sedentarismo & 55 & 42 & $<0,0001$ \\
\hline Diabetes & 24 & 20 & 0,026 \\
\hline Obesidade & 22 & 10 & $<0,0001$ \\
\hline Tabagismo & 9 & 11 & 0,10 \\
\hline Doença vascular periférica & 1 & 2 & 0,51 \\
\hline$>2$ fatores de risco & 63 & 41 & $<0,0001$ \\
\hline
\end{tabular}

Teste T de Student para amostras independentes ou o Teste de Mann-Whitney.
Tabela 3 - Prevalência dos fatores de risco por faixa etária.

\begin{tabular}{lccc}
\hline & $\begin{array}{c}\text { Idade }<55 \\
\text { anos } \%\end{array}$ & $\begin{array}{c}\text { Idade }>65 \\
\text { anos } \%\end{array}$ & \\
\hline História Familiar & 58 & 46 & 0,005 \\
\hline Ex-tabagismo & 56 & 46 & 0,028 \\
\hline Obesidade & 15 & 8 & 0,008 \\
\hline Tabagismo & 15 & 5 & $<0,0001$ \\
\hline Hipertensão & 56 & 56 & 0,93 \\
\hline Dislipidemia & 51 & 52 & 0,77 \\
\hline Sedentarismo & 47 & 42 & 0,45 \\
\hline Diabetes & 20 & 22 & 0,59 \\
\hline Doença vascular periférica & 3 & 2 & poucos casos \\
\hline$>2$ fatores de risco & 51 & 42 & 0,049 \\
\hline
\end{tabular}

Teste T de Student para amostras independentes ou o teste de Mann-Whitney. 


\section{Ponto de vista}

Quando analisados estes dados em conjunto, fica a impressão de que os adultos de menor poder econômico apresentam maior risco cardiovascular pela maior prevalência de $F R$, o que não ocorre na infância, podendo-se concluir que, neste caso, uma melhor informação/educação poderia influenciar e modificar este quadro. Com relação ao sexo e à idade, parece ser mais fácil a identificação dos maiores candidatos a desenvolver aterosclerose, pelo maior acúmulo de FR nas mulheres e nos mais jovens. Cabe lembrar que estes são os grupos que costumam ter pior evolução quando apresentam DAC.

Estudos mostram os beneficios do controle dos FR, como o estudo de Ciorlia e Godoy ${ }^{13}$, com segmento de ate 20 anos. Neste contexto, podemos concluir que existe uma grande possibilidade de modificação na incidência e talvez na mortalidade por DAC, a partir de campanhas de

\section{Referências}

1. Ford ES, Ajani UA, Croft JB, Critchley JA, Labarthe DR, Kottke TE, et al. Explaining the decrease in U.S. deaths from coronary disease, 1980-2000. N Engl J Med. 2007; 356: 2388-98.

2. Piegas LS, Avezum A, Pereira JC, Neto JM, Hoepfner C, Farran JA, et al. AFIRMAR Study Investigators. Risk factors for myocardial infarction in Brazil. Am Heart J. 2003; 146: 331-8.

3. McGill HC Jr, McMahan CA Gidding SS. Preventing heart disease in the 21st century: implications of the Pathobiological Determinants of Atherosclerosis in Youth (PDAY) study. Circulation. 2008; 117: 1216-27.

4. Ministério da Saúde. Datasus: informações de saúde. [Acesso em 2008 Nov 25]. Disponível em: http://tabnet.datasus.gov.br/cgi/tabcgi.exe?idb2007/c04.def

5. Laurenti R, Buchalla CM. Os mitos a respeito das doenças cardiovasculares. Arq Bras Cardiol. 2001; 76: 99-110.

6. Ribeiro RA, Mello RG, Melchior R, Dill JC, Hohmann CB, Lucchese AM, et al. Annual cost of ischemic heart disease in Brazil: public and private perspective. Arq Bras Cardiol. 2005; 85: 3-8.

7. Scherr C, Magalhães CK, Malheiros W. Lipid profile analysis in school children. Arq Bras Cardiol. 2007; 89: 65-70. esclarecimento e prevenção, como as existentes para câncer de mama, dengue e AIDS.

\section{Potencial Conflito de Interesses}

Declaro não haver conflito de interesses pertinentes.

\section{Fontes de Financiamento}

O presente estudo não teve fontes de financiamento externas.

\section{Vinculação Acadêmica}

Não há vinculação deste estudo a programas de pósgraduação.

8. Scherr C, Magalhães CK, Silva LJD, Anello A, Sá MR. Fatores de risco para coronariopatia no sexo feminino. Arq Bras Cardiol. 2000; 74 (supl 1): 25.

9. Scherr C, Loyola LH, Rivas MBS, Ferreira MF. Análise da prevalencia dos fatores de risco em adultos jovens coronariopatas: estudo retrospectivo. Rev SOCERJ. 1997; 10 (supl A): 13.

10. Scherr C, Pontes PV, Alves PM. Cardiopatia Isquêmica até os 45 anos: análise da prevalência de fatores de risco coronariano. Arq Bras Cardiol. 1992; 59 (supl I): 125

11. Scherr C, Abitibol RA, Noe R. Fatores de risco para doença coronariana - um problema ainda não solucionado. Rev Soc Cardiol Estado de São Paulo. 2005; 15: 94.

12. Scherr C. Impacto de um programa de controle de fatores de risco para doença arterial coronária obstrutiva. São Paulo: Editora Best Point; 2005.

13. Ciorlia LA, Godoy MF. Cardiovascular risk factors and mortality: longterm follow-up (up to 20 years) in a preventive program carried out by occupational medicine. Arq Bras Cardiol. 2005; 85: 20-5. 